\title{
Visualization of the power waveforms frequency fluctuations with the use of the constant length time window
}

\author{
J. Zygarlicki ${ }^{1}, \mathrm{~J}_{\text {. Mroczka }}{ }^{2}$ \\ ${ }^{1}$ Faculty of Electrical Engineering, Automatic Control and Informatics \\ Opole University of Technology \\ ul. Proszkowska 76, 45-758 Opole (Poland) \\ Phone/Fax number: +0048 77 4000547, e-mail: j.zygarlicki@ po.opole.pl \\ ${ }^{2}$ Faculty of Electronics Wroclaw University of Technology \\ ul. B. Prusa 53/55, 50-317 Wroclaw (Poland) \\ Phone/Fax number: +0048 71 3206232, e-mail: janusz.mroczka@pwr.wroc.pl
}

\begin{abstract}
This paper presents a new method of visualization of the power waveforms frequency fluctuations in terms of analysis of harmonics and interharmonics in an electric power signal. The presented method is based on image analysis and can be competitive, in some specific cases, to both Fourier transformation and wavelet method. The presented method constitutes a specific image preparation which consists of a split waveform signal. The article shows examples of simulations results for real life and simulated signals.
\end{abstract}

\section{Key words}

Power quality, harmonics, Fourier transform, signal processing, image analysis

\section{Introduction}

Recently, due to the considerable increase in the number of power receivers as well as gradual liberalization of the power market, more attention has been paid to the quality of power. Knowledge of the performance of the power system and the quality of the power signal sent enables modernization and installation of additional protection for usually expensive electric and electronic devices. Although there are plenty of different devices for online analysis and monitoring of power quality nowadays, post event processing is still important. It enables advanced feature extraction for detection, localization and characterization of PQ events.

Several methods using Fourier transform, wavelet, Gabor, Prony's method or Wigner-Ville distribution have been developed for analysis of power quality, in e.g.: [1]-[5]. In this paper, the authors develop two-dimensional (2D) representation of the power quality signal introduced in [6]. Presented method enables both variability of power waveform frequency analysis and detection of impulse and transient events, because prepared 2D representation of the signal has more operation flexibility than the regular 1D representation. In Section 2, presented method of power waveform analysis is developed. A combination of image analysis, Fourier transformation and least squares method is proposed as a tool to apply on the $2 \mathrm{D}$ data. To test the method's performances, the authors used voltage waveforms of simulated and also real life power quality events. In Section 3 the analysis results are obtained using the method described in Section 2. It is observed that the presented method easily detects such power quality event types as frequency fluctuations, but also sags, swells, impulses and oscillatory transients.

\section{Method description}

Figure 1 illustrates the method of a 1D signal transformation to a 2D image. The new representation of power waveform signal consists of two-dimensional matrix whose rows correspond to prepared segments of 1D signal. The columns of that matrix represent the numbers of the time windows. Prepared 2D image can be analyzed in the next step with the known image processing methods, Least Squares estimation method and Fourier transformation.

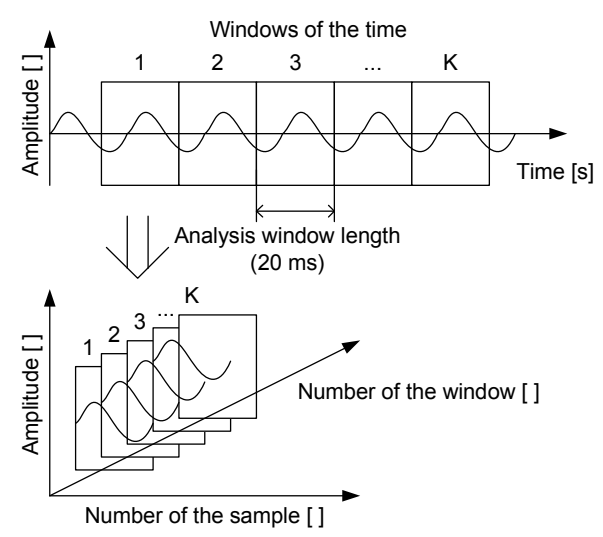

Fig. 1. An image (2D) preparation from regular 1D signal representation.

Figure 2 illustrates the process of finding fundamental harmonic, constructing vector of data - edge in an image that is then used for fundamental frequency estimation and computation of its fluctuations in time. The first step in this block scheme is binarization of the image, next edge detection with image processing method and then, again, another binarization. After these steps, the longest of the edges found is transformed to frequency vector 
that makes function of time. Derivative of the vector is then computed:

$$
d y=\left[\begin{array}{llll}
y_{2} & y_{3} & \ldots & y_{N}
\end{array}\right]^{\prime}-\left[\begin{array}{llll}
y_{1} & y_{2} & \ldots & y_{N-1}
\end{array}\right]^{\prime}
$$

where: $y_{\mathrm{n}}-$ time of the signal sample of the edge, $N$ - length of the founded edge.

The Least Squares method is used for approximation of fundamental frequency fluctuations by means of linear equation:

$$
\hat{f}=X\left(\left(X^{\prime} X\right)^{-1} X^{\prime} f\right)
$$

where:

$$
\begin{aligned}
& \hat{f}=\left[\begin{array}{llll}
\hat{f}_{1} & \hat{f}_{2} & \ldots & \hat{f}_{N-1}
\end{array}\right], X=\left[\begin{array}{cccc}
t_{1} & t_{2} & \ldots & t_{N-1} \\
1 & 1 & \ldots & 1
\end{array}\right]^{\prime}, \\
& f=\left[\begin{array}{llll}
\frac{1}{T+d y_{1}} & \frac{1}{T+d y_{2}} & \cdots & \frac{1}{T+d y_{N-1}}
\end{array}\right]^{\prime},
\end{aligned}
$$

$\hat{f}$ - approximation of fundamental frequency fluctuations, $f$ - fundamental frequency fluctuations, $t_{\mathrm{n}}$ - time of the $\mathrm{n}^{\text {th }}$ window, $T$ - length of the analysis window $(20 \mathrm{~ms})$.

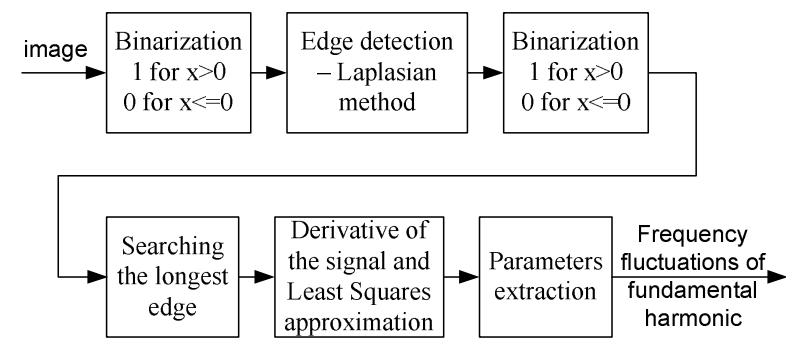

Fig. 2. Frequency fluctuations of fundamental harmonic computation using image analysis methods.

Figure 3 illustrates edge detection with Laplasian mask [7]. Each new pixel of the analyzed image is computed as a sum of the product of Laplasian mask and pixels corresponding in the image.

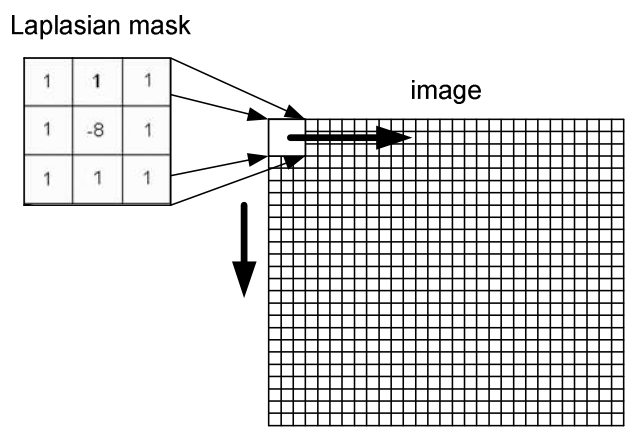

Fig. 3. An image edge detection using Laplasian mask.

Figure 4 illustrates the method of eliminating fundamental harmonic used for presentation of selected events. The presented method is a kind of filtration where the analyzed signal is first transformed to frequency domain with Fourier method and then the components of frequency of $50 \pm 25 \mathrm{~Hz}$ are set to zero. The last step is computation of inverse Fourier transform.

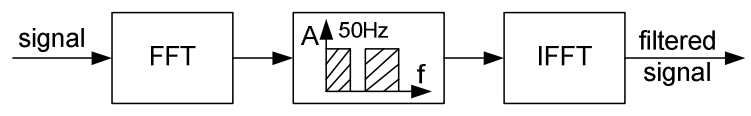

Fig. 4. Elimination of fundamental harmonic from 1D signal for $2 \mathrm{D}$ visualizations of transients and impulses.

\section{Results}

The simulations have been made for real life and simulated signals. First test signal only consists of the fundamental harmonic (figures 5-8). The frequency of fundamental harmonics has grown up in time window from $49 \mathrm{~Hz}$ to $51 \mathrm{~Hz}$. Figure 7 shows zero-crossing detection done with image processing from the simulated 2D signal representation. Next, the results were used for the Least Squares estimation for computation of the fundamental harmonic fluctuations.

Next test signal consists of the fundamental harmonic of constant frequency: $49.9 \mathrm{~Hz}$ (figures 9-11).

The next simulations were made for real signals recorded with Elspec G4430 BlackBox with sampling frequency of $12.8 \mathrm{kHz}$ and resolution of 16 bits. Figures 12-14 illustrate the recorded impulse event, figures 15-18 show results for dip event and figures 19-21 demonstrate results for normal signals with no events. Selected figures show events with eliminated fundamental harmonic for improving visualization.

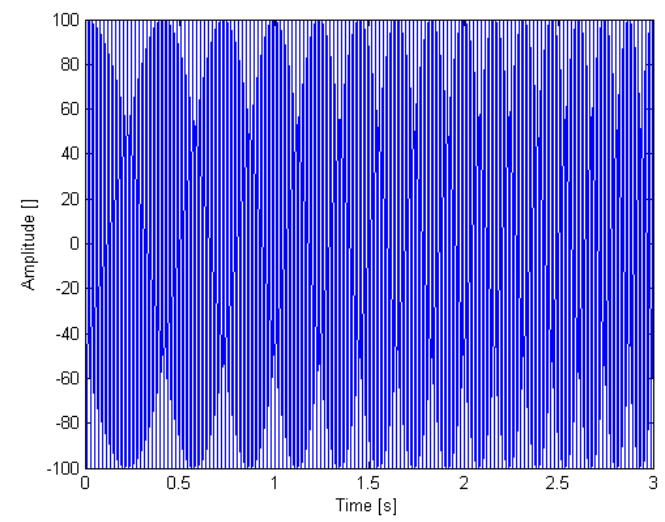

Fig. 5. The simulated 1D signal only consists of the fundamental harmonic. The frequency of fundamental harmonics has grown up in the presented window from $49 \mathrm{~Hz}$ to $51 \mathrm{~Hz}$.

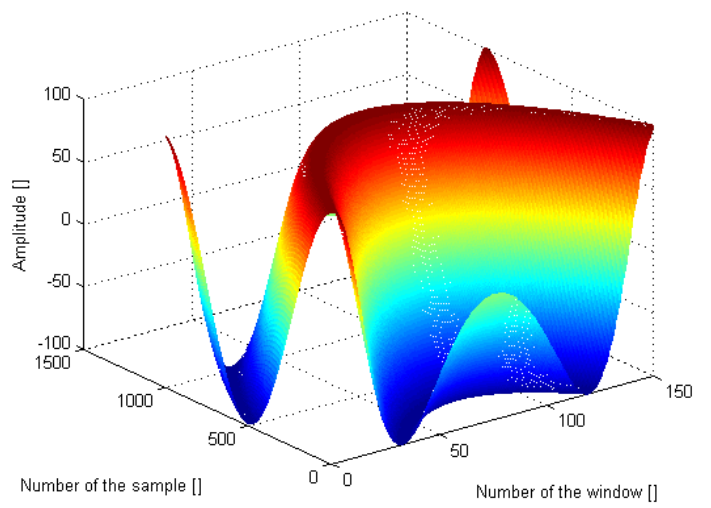

Fig. 6. The simulated 2D signal representation only consists of the fundamental harmonic (figure 5). The frequency of fundamental harmonic has grown up in presented window from $49 \mathrm{~Hz}$ to $51 \mathrm{~Hz}$. 


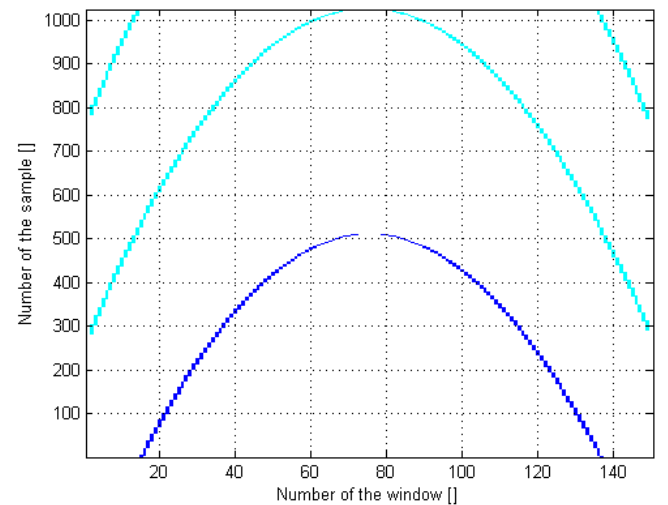

Fig. 7. Zero-crossing detection done with image processing from the simulated 2D signal representation (figure 6).

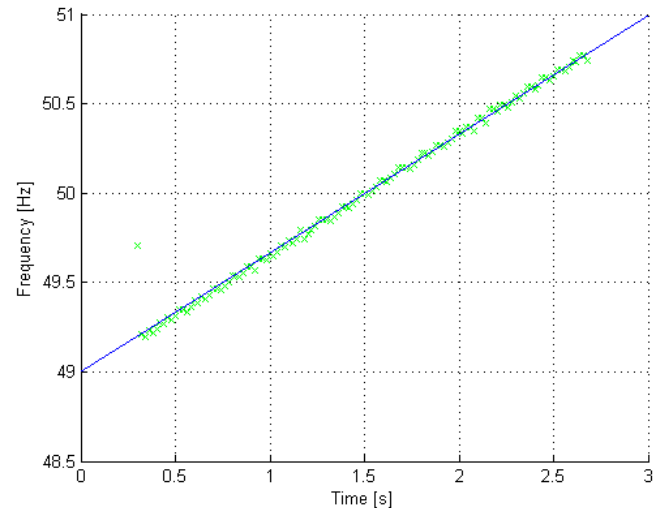

Fig. 8. Least Squares estimation of the fundamental harmonic fluctuations from figure 7 .

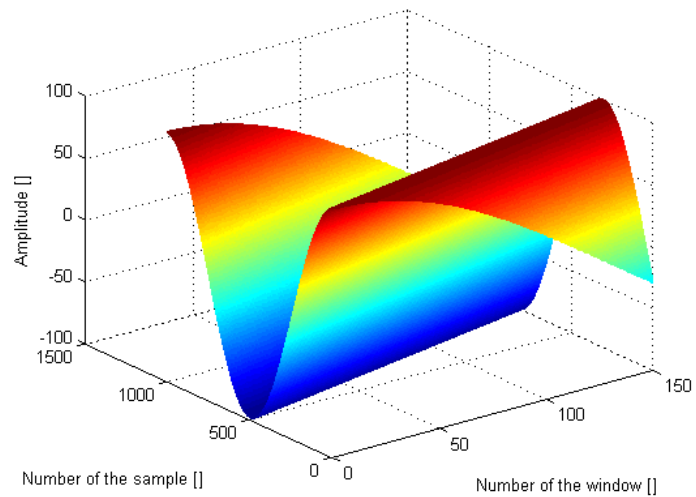

Fig. 9. The simulated 2D signal representation only consists of the fundamental harmonic. The frequency of fundamental harmonic is constant: $49.9 \mathrm{~Hz}$.

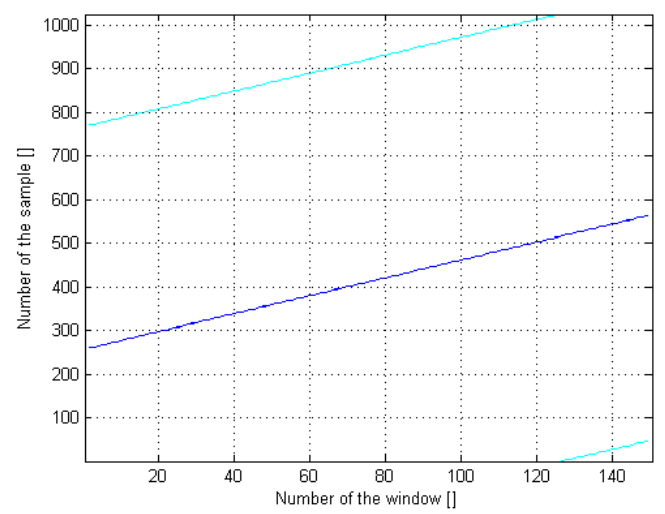

Fig. 10. Zero-crossing detection done with image processing from the simulated 2D signal representation (figure 9).

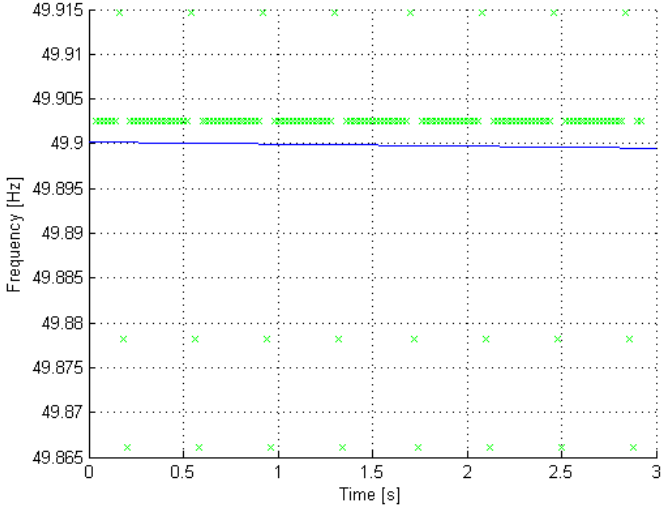

Fig. 11. Least Squares estimation of the fundamental harmonic fluctuations from figure 10 .

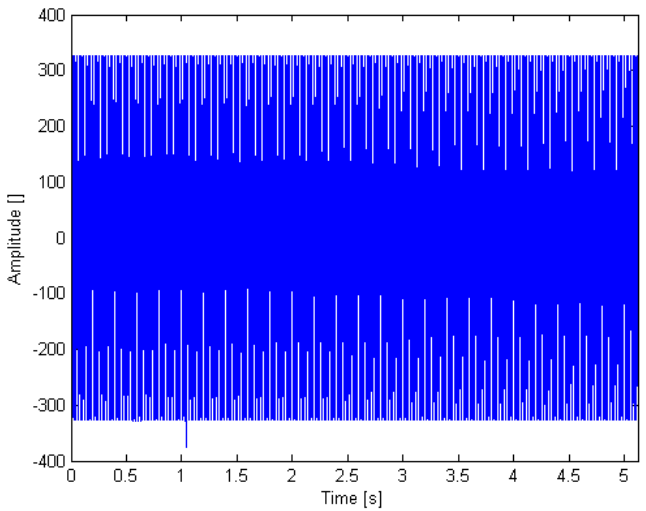

Fig. 12. The real 1D signal with impulse event.

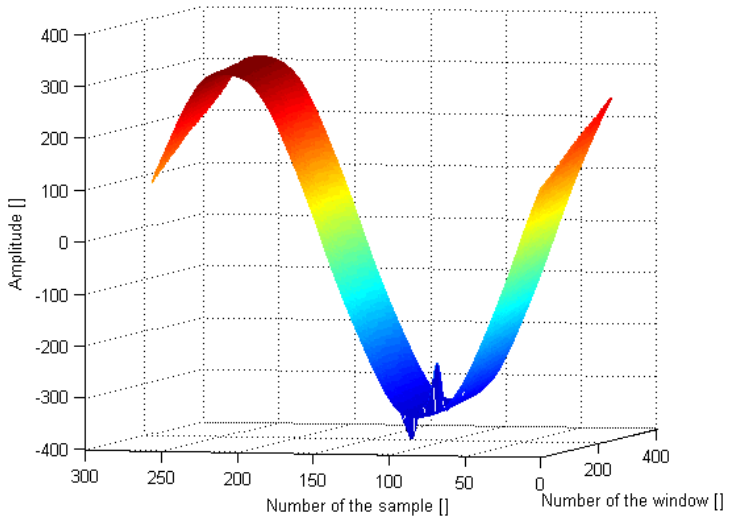

Fig. 13. The real 2D signal representation with impulse event from figure 12 .

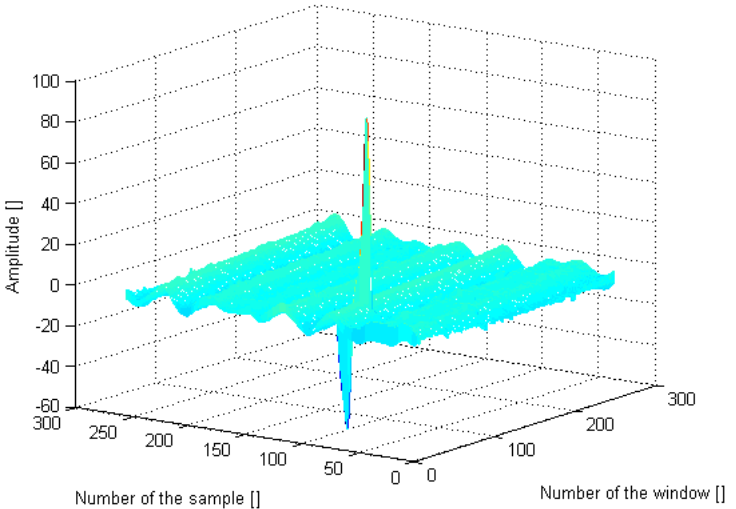

Fig. 14. The real 2D signal representation with impulse event from figure 12. Fundamental harmonic eliminated according to figure 4. 


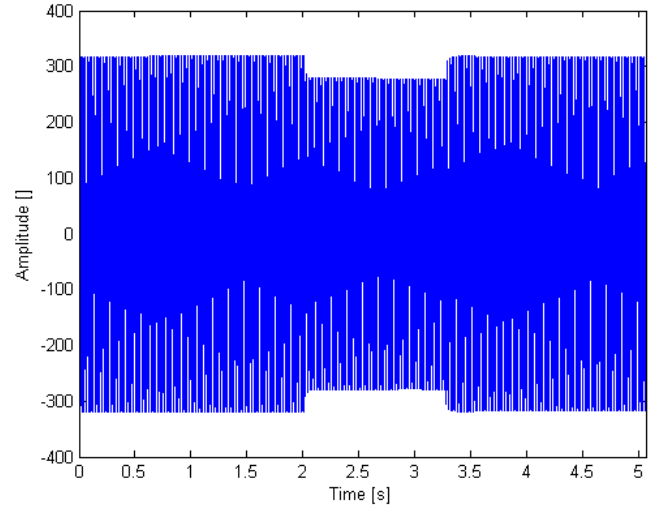

Fig. 15. The real 1D signal with dip event.

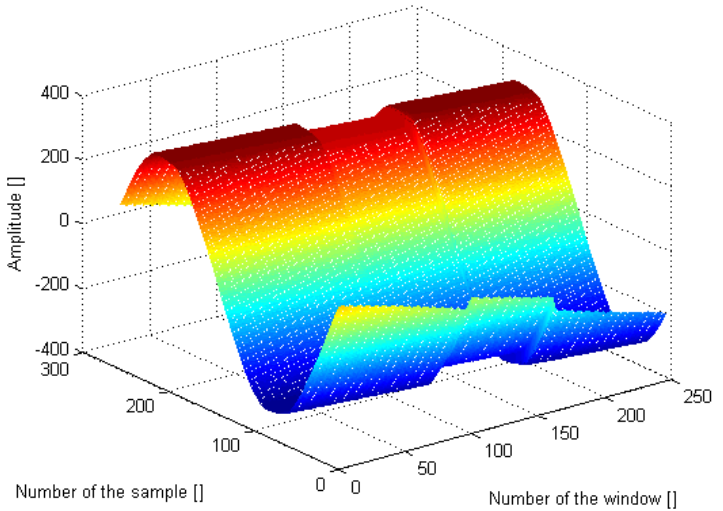

Fig. 16. The real 2D signal representation with dip event from figure 15

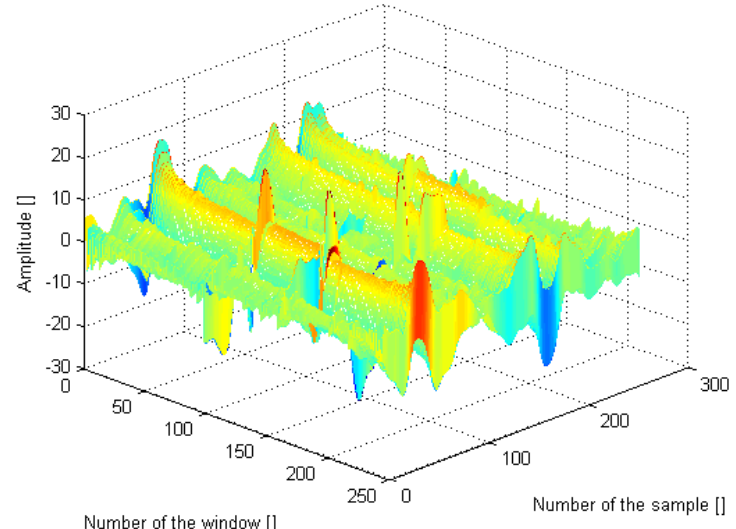

Fig. 17. The real 2D signal representation with dip event from figure 15. Fundamental harmonic eliminated according to fig. 4.

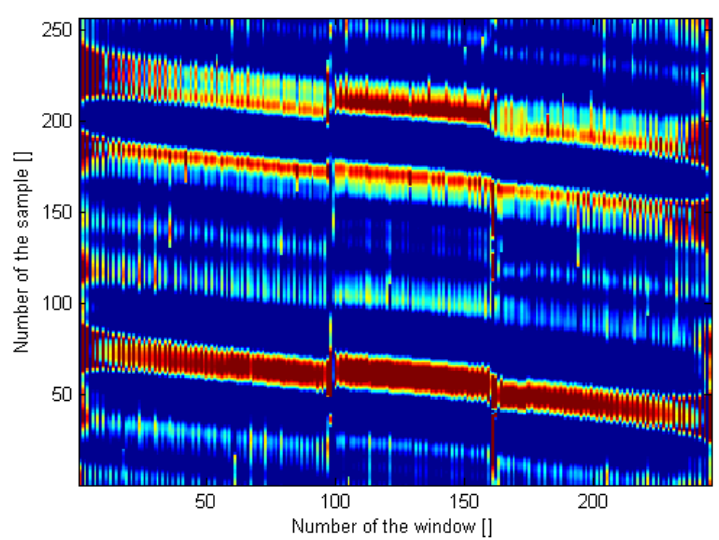

Fig. 18. The real 2D signal representation with dip event from figure 15. Fundamental harmonic eliminated according to fig. 4 top view.

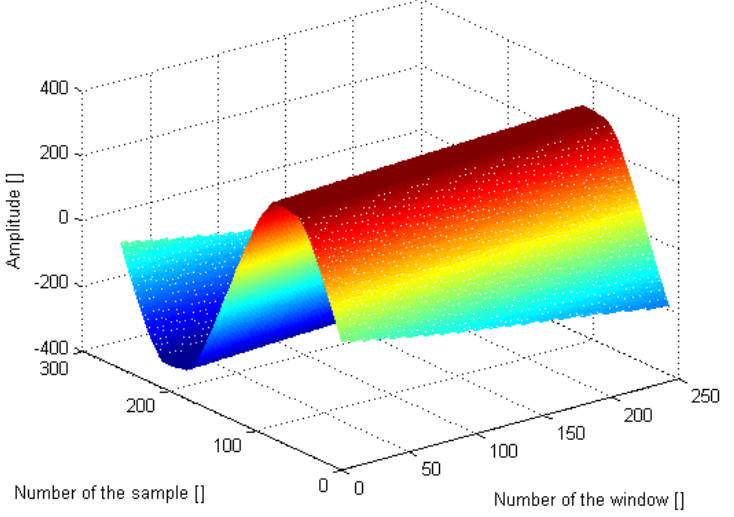

Fig. 19. The real 2D signal representation with no registered events.

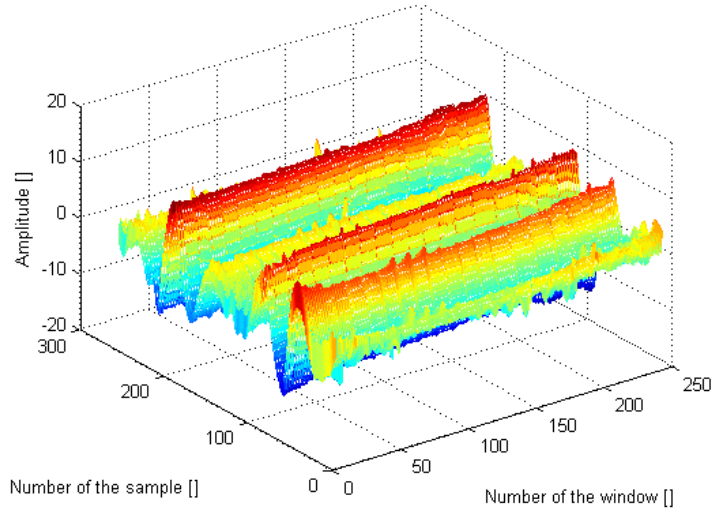

Fig. 20. The real 2D signal representation with no registered events. Fundamental harmonic eliminated according to figure 4.

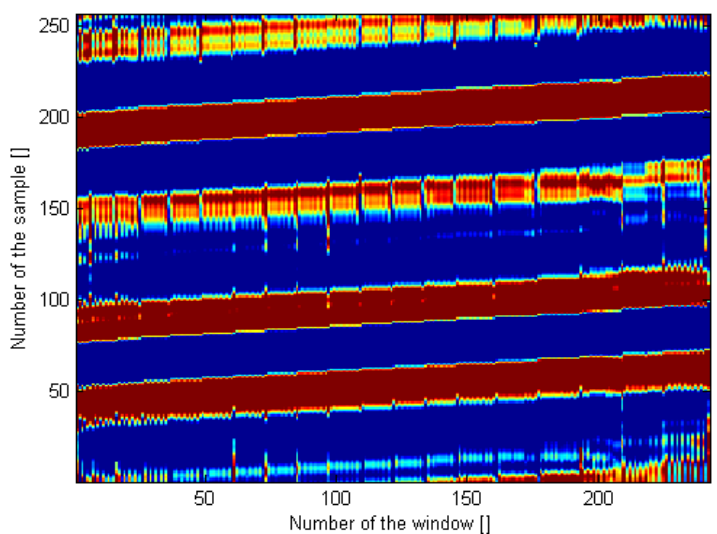

Fig. 21. The real 2D signal representation with no registered events. Fundamental harmonic eliminated according to fig. 4 - top view.

\section{Conclusions}

In this work a $2 \mathrm{D}$ representation of power waveforms is developed. The method proposed can be used for an easy analysis of fluctuations of harmonics as well as interharmonics and subharmonics in an electric power signal.

The presented method also enables detection and visualization of interharmonics and subharmonics that are not synchronized with fundamental frequency.

It is observed that the presented method easily detects such power quality event types as frequency fluctuations, but also sags, swells, impulses and oscillatory transients. 
The presented method, based on image analysis, can be competitive in specific cases to both Fourier transformation method and wavelet method and can be a useful tool for advanced power waveform analysis that goes beyond actual specification included in the standards related to power quality.

\section{Acknowledgement}

This work was supported by the Ministry of Science and Higher Education, Poland (grant \# N N505 557439).

\section{References}

[1] J. Zygarlicki, M. Zygarlicka, J. Mroczka, K. Latawiec, “A reduced Prony's method in power quality analysis - parameters selection”, IEEE Transactions on Power Delivery, vol. 25, no. 2, April 2010.

[2] T. Lobos, Z. Leonowicz, J. Rezmer, P. Schegner, "Highresolution spectrum-estimation methods for signal analysis in power systems", IEEE Trans. Instr. and Meas., vol. 55, no. 1, pp. 219-225, Feb. 2006.

[3] M. Szmajda, K. Górecki, J. Mroczka, "Gabor Transform, Gabor-Wigner Transform and SPWVD as a Time-Frequency Analysis of Power Quality", 14th International Conference on Harmonics and Quality of Power, Bergamo (Italy) 26-29 September 2010.

[4] K. Gorecki, M. Szmajda, J. Mroczka, "Mixed algorithms radix in harmonics measurements of low-voltage power networks", Proceedings of the 9th International Scientific Conference - Electric Power Engineering EPE2008, s. 121-124.

[5] T. Tarasiuk, "Hybrid Wavelet-Fourier Method for Harmonics and Harmonic Subgroups Measurement-Case Study", IEEE Trans. Power Del., vol. 22, no. 1, pp. 4-17, Jan. 2007.

[6] O.N. Gerek, , D.G. Ece, , "A 2D representation for analysis and coding of power quality events", International Conference on Image Processing, ICIP 2003.

[7] L. Wojnar, M. Majorek, Komputerowa analiza obrazu, Computer Scaning Systems Sp. z o. o., Warszawa 1994. 